\title{
L'aspect de phase en français: le rôle des périphrases verbales
}

\section{LAURENT GOSSELIN}

Université de Rouen

(Received March 2009; revised August 2009)

\section{RÉ S U M É}

Cet article porte sur les périphrases aspectuelles (commencer à, être en train de, etc.). On montre que si les grammaires divergent fortement à propos du statut lexical ou grammatical qu'il convient de leur accorder, il est néanmoins possible et pertinent de dissocier deux classes de périphrases, auxquelles on attribue un fonctionnement sémantique nettement différent. Les unes servent à construire un sous-procès, tandis que les autres indiquent un type de visée aspectuelle particulier. Cette analyse prend appui sur des études récentes (Kronning, François, Laca), qui montrent de façon indépendante, mais convergente, qu'il est nécessaire de dissocier deux classes de périphrases aspectuelles sur la base de leur comportement syntaxique.

\section{LA STRUCTURE PHASALE DES PROCÈS}

Nous considérons 'l'aspect de phase', sous lequel est présenté un procès (état ou événement), comme le résultat d'une opération de sélection d'une partie (phase) du temps constitutif de ce procès. Cette opération est nécessairement complémentaire du repérage temporel, car, comme l'indiquait clairement Brunot dès I922, ce n'est pas le procès pris globalement qui se trouve temporellement localisé, mais seulement la partie qui en est sélectionnée:

[...] le temps ne peut être complètement indiqué que si on exprime, d'une part, à quel moment se rapporte l'action, d'autre part, à quel point de son développement elle en est à ce moment. [...] Supposons qu'on donne rendez-vous à quelqu'un qui vous a présenté un manuscrit: revenez lundi, je l'aurai $\mathbf{l u}$, nous en causerons. Ce qu'on veut lui marquer par je l'aurai lu, c'est que ce jour-là la lecture sera terminée, le fait accompli. L'action se présentera sous l'aspect d'une action accomplie.' (Brunot, I922: 440).

Pour autant, ce temps constitutif ('impliqué' selon Guillaume I964: 47, 'présupposé par le procès' selon Barcelo et Bres, 2006: I2) ne se limite pas - contrairement à ce qu'une formulation approximative laisse généralement entendre au temps 'interne' du procès (i.e. compris entre ses bornes initiale et finale), mais englobe aussi les phases préparatoire et résultante du procès, ce que Borillo (2005: 67) nomme 'l'aspect externe', par opposition à 'l'aspect interne'. Si l'on admet le découpage classique de l'aspect interne en trois phases (début, milieu, fin) 
auquel s'ajoutent les deux phases 'périphériques' de l'aspect externe, on obtient une structure méréotopologique (i.e. qui concerne la disposition des parties à l'intérieur du tout, cf. Cazatti et Varzi, I999) organisée en cinq phases virtuellement sélectionnables, et illustrée par la Figure I (voir aussi Dik, I989: I90).

B1

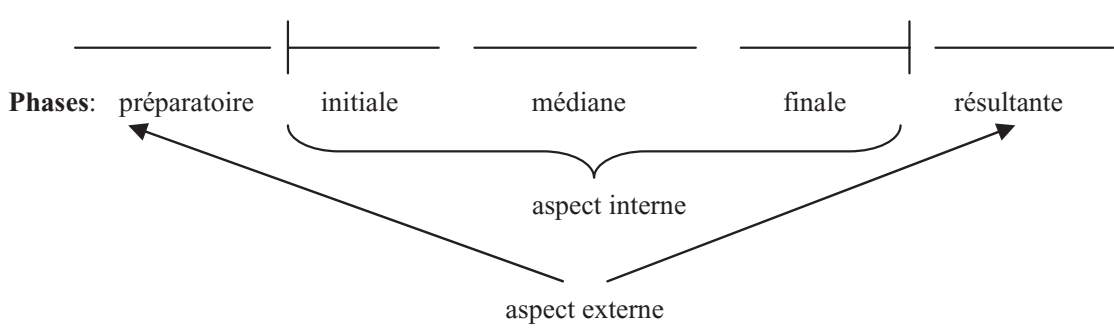

Figure I. La structure phasale des procès.

Nous admettons que les phases initiale et finale contiennent respectivement les bornes initiale et finale du procès sans pour autant s'y réduire (ce qui distingue notre analyse de celle de Vet, 2002). Cela nous permettra d'expliquer naturellement la compatibilité de 'commencer à Vinf et de 'finir de Vinf avec la visée aspectuelle imperfective: l'expression 'être en train de finir de manger' indique précisément que la phase finale est 'en cours' et donc que la borne finale du procès, quoique proche, n'est pas encore atteinte (voir ci-dessous, $§ 6$ ).

Cette structure phasale paraît valoir pour tout type de procès (y compris les séries itératives), à l'exception des états nécessaires (qui n'ont ni commencement ni fin), et relever de la linguistique générale. Comme l'affirme Tournadre (2004: 23):

'Il est possible de sélectionner une phase d'un procès pour en faire l'objet de la prédication. En théorie, chaque procès offre la possibilité de choisir parmi cinq phases: pré-processuelle, initiale, médiane, finale, post-processuelle.'

Car même s'il est vrai qu'un procès ponctuel ne laisse pas observer son aspect interne, toute forme de dilatation de ces procès manifeste - en même temps qu'elle leur fait perdre leur ponctualité - leur décomposition virtuelle en trois phases internes. Hugo affectionne particulièrement ces 'grossissements fantastiques' (voir la dilatation du procès 'pousser la porte' dans Les Misérables, première partie, II, I I, cité dans Gosselin, I996: 68-69, ou celle de 'jeter le livre à terre' dans Quatrevingt-treize, III, 3).

Prenons garde toutefois à ne pas mettre sur le même plan cette structure phasale qui concerne le procès une fois qu'il est catégorisé, avec les figures qui rendent compte de l'opération de catégorisation à partir d'un schéma primitif (pré-conceptuel) comportant des changements et des situations intermédiaires, comme celles qui sont proposées par Moens et Steedman (I988), Kamp et Reyle (I993: 558 sq.), Gosselin (I996: 50 sq.), ou encore Croft (en préparation). Car dans 
ce cas, la portion du schéma primitif subsumée par la catégorie de procès varie systématiquement en fonction du type de procès (état, activité, accomplissement, achèvement). Un exemple nous servira à illustrer la différence radicale de niveau d'analyse. Les états transitoires correspondent au plan du schéma primitif à une absence de changement (ils sont donc adéquatement représentés par un segment de droite, dont les bornes sont exclues, cf. Kamp et Reyle, I993: 562, Gosselin, I996: 54, Croft en prép. $\S$ I.2.2.), mais une fois l'état catégorisé, c'est-à-dire une fois que ce segment de droite se trouve subsumé par une catégorie, (ex. être malade), la structure phasale commune s'impose: on peut alors sélectionner une phase préparatoire ('je vois bien qu'il va être malade'), initiale ('tomber/ commencer à être malade'), médiane (continuer d'être malade), finale ('cesser d'être malade'), et résultante ('on voit bien qu'il vient d'être malade'). On distinguera donc soigneusement deux niveaux d'analyse de l'aspect lexical: un niveau pré-conceptuel et un niveau conceptuel. La structure phasale commune, décrite par la Figure I, ne vaut qu'au niveau conceptuel, i.e. une fois l'opération de catégorisation (profilage) réalisée.

\section{COMMENT SÉLECTIONNER LES PHASES D'Un PROCÈs EN FRANÇAIS?}

Poser la question de l'aspect de phase revient donc à se demander comment on peut rendre saillantes dans l'énoncé ces phases latente, ou encore, pour reprendre les termes de Brunot, comment on peut sélectionner une phase ('un point de développement') du procès, pour la mettre en relation avec un repère temporel?

A la suite d'une investigation de grande ampleur, Tournadre (2004: 23) observe que 'la sélection [d'une phase] est susceptible d'être réalisée selon les langues ou les classes verbales par des moyens lexicaux ou grammaticaux'. Appliquée au français, la question devient alors de savoir par quels moyens, lexicaux et/ou grammaticaux, cette langue permet la sélection d'une ou plusieurs phase(s) d'un procès. Cependant cette question ne concerne pas seulement le statut morphologique (lexèmes ou grammèmes?) des marqueurs de phase, mais plus fondamentalement la nature des opérations sémantiques déclenchées par ces marqueurs.

Traditionnellement, pour un procès donné (exprimé par une prédication complète), on considère qu'il existe deux moyens principaux en français: les conjugaisons (temps simples et composés) et les périphrases verbales (constituées typiquement d'un semi-auxiliaire ou coverbe - terme utilisé par Roy, I976, Wilmet, I996 et Kronning, 2003 - suivi d'un verbe à l'infinitif). A quoi, on peut ajouter d'une part certains préfixes, comme en qui est tenu pour inchoatif dans s'endormir ou s'envoler (Martin I97I: 53) ainsi que les 'verbes supports aspectuels' (cf. Borillo 2006) qui permettent également de sélectionner la phase d'un procès (ex.: être en larmes $\rightarrow$ fondre en larmes, faire une recherche $\rightarrow$ entamer / poursuivre une recherche), et d'autre part le fait que la présence (sous certaines conditions) d'un circonstanciel temporel normalement incompatible avec la structure intrinsèque du procès puisse entraîner un glissement de sens consistant à retenir l'une des phases de ce dernier (celle qui est compatible avec le circonstanciel; cf. Gosselin, I996: 56-63). C'est 
ainsi que dans 'dormir à huit heures', le circonstanciel ponctuel, entraîne un glissement vers la phase initiale ponctuelle du procès qui est intrinsèquement non ponctuel (l'expression devient équivalente à 's'endormir à huit heures'). Et inversement, la présence d'un circonstanciel de durée portant sur un procès intrinsèquement ponctuel peut conduire à un glissement vers la phase préparatoire (ex. 'il s'arrêta en dix secondes') ou vers la phase résultante du procès (ex. 'il s'arrêta pendant dix secondes', cf. Borillo, I986: I38-I39). Nous nous attacherons, dans cet article à définir le statut et le rôle sémantique des périphrases verbales.

Alors que les conjugaisons relèvent de l'aspect grammatical, la question se pose du statut lexical ou grammatical des périphrases verbales. Les grammaires et ouvrages généraux sur la temporalité en français divergent fortement sur ce point. Les positions les plus communément adoptées consistent soit à considérer que toutes les périphrases verbales relèvent de l'aspect lexical, par opposition aux conjugaisons (voir par exemple Wagner et Pinchon, I962: 298), soit à intégrer [aller Vinf] et parfois [venir de Vinf] aux conjugaisons (et donc à l'aspect grammatical), tandis que les autres périphrases seraient de nature lexicale. L'argument souvent avancé (voir, entre autres, Laca, 2004: 90, Vet, 2008: 458) en faveur de cette option est que ces périphrases, à la différence des autres, ne tolèrent pas toutes les conjugaisons. Ce critère paraît cependant bien difficile à mettre en œuvre: faut-il intégrer 'venir de' qui accepte le futur, et 'être en train de' qui tolère même le passé simple et les temps composés dans certains contextes (ex. 'Dès qu'ils furent / ont été en train de manger')? Et par ailleurs, son application devrait logiquement conduire à refuser le statut d'auxiliaire aspectuel à être et avoir! C'est précisément pourquoi ce même critère avait conduit Martin (I97I: I40) à exclure aller et venir du système des temps du français. Deux analyses plus radicales, ont été proposées: Wilmet (I997: 3 I6) intègre à la fois la totalité des périphrases verbales, mais aussi les temps composés (aux + participe passé) à l'aspect lexical, qu'il oppose non plus à l'aspect grammatical, mais à l'aspect affixal, caractéristique des temps simples. A l'inverse, Leeman-Bouix (I994: 5I) ainsi que Barcelo et Bres (2006: I6) paraissent considérer que toutes les périphrases verbales relèvent de l'aspect grammatical.

A l'origine de ces difficultés, il y a tout d'abord le fait que les auxiliaires et les affixes verbaux résultent d'un processus continu de grammaticalisation que l'on a pu représenter au moyen de la Figure 2.

$$
\text { Verbe plein }>\mathrm{AUX}_{1}>\ldots \mathrm{AUX}_{\mathrm{n}}>\text { Affixe }
$$

Figure 2. Le parcours de grammaticalisation des verbes ('The Verb-to-TAM Chain', Heine, 1993, Hopper et Traugott, 1993, Kronning, 2003).

Il apparait alors relativement arbitraire de tracer une frontière précise entre lexèmes et grammèmes sur un tel continuum et de répartir les différentes expressions aspectuelles de part et d'autre de cette frontière, ce que reconnaissent Arrivé et al. (I986: 9I: 'On est ici aux confins des structures grammaticales et lexicales'), Riegel et al (I994: 253; 'Si certains [de ces auxiliaires] ont indiscutablement un statut 
comparable à celui d'avoir et être, d'autres possèdent un sens lexical qui les rapproche plutôt d'un verbe ordinaire'), et de façon plus explicite encore Lagae, Carlier et Benninger (2002 : i):

Même à l'intérieur d'une même langue, on ne peut admettre l'existence d'une réelle frontière entre grammaire et lexique. En effet, certains marqueurs temporels ou aspectuels se trouvent dans la zone frontière entre grammaire et lexique. Il en va ainsi des semi-auxiliaires d'aspect du français: leur origine étant lexicale, ils ont subi un processus de grammaticalisation qui n'a toutefois pas entièrement abouti.

La situation se complique singulièrement si l'on considère qu'en fonction des critères (syntaxiques et sémantiques) retenus, tel ou tel coverbe présentera des 'degrés de verbalité' différents, de sorte qu'il paraît presque impossible de les ordonner sur un même et unique continuum (comme le montre BlancheBenveniste, 200I).

De plus, il ne semble pas que l'on puisse déduire directement la nature de la contribution sémantique d'une périphrase verbale à l'énoncé, en se fondant sur sa position sur l'échelle de grammaticalisation. Deux raisons, au moins s'y opposent:

a) Le lien entre grammaticalisation et valeur sémantique est généralement décrit en termes de perte de traits sémantiques (désémantisation, usure sémantique, 'javellisation'). Or cette analyse suppose, pour prendre un contenu précis, une conception quantitative, quasi arithmétique, de la sémantique (de sorte qu'il soit envisageable d'effectuer des soustractions dans le domaine du sens), laquelle n'est guère compatible avec les théories actuelles du temps et de l'aspect (qui ne considèrent généralement pas le sens des marqueurs d'aspect comme de simples collections de traits sémantiques).

b) Tournadre (2004: 34) met en cause la distinction même entre aspect lexical et aspect grammatical, considérant que le choix du type de marqueur (lexical ou grammatical) ne correspond pas nécessairement à une différence sémantique profonde, car il montre, exemples à l'appui, que 'l'aspect grammatical d'une langue peut correspondre à un aspect lexical dans une autre langue'. Au fond, il se pourrait que la question telle que nous l'avons posée (dans le cadre de la tradition de la grammaire française) relève d'une 'approche erronée' (Tournadre, 2004: 34), qui ne fait pas nettement le départ entre la nature morpho-syntaxique des catégories utilisées et les opérations sémantiques qu'elles codent. De là, la nécessité de poser le problème de la sélection des phases d'un procès à nouveaux frais, dans un cadre théorique explicite.

\section{L'ASPECT, ENTRE CATÉgorisation ET MONSTRATION}

On admet, suivant le modèle présenté dans Gosselin (I996 et 2005) et nommé désormais modèle SdT (pour Sémantique de la Temporalité, cf. Person, 2004, Gosselin et Person, 2005), que l'aspect peut résulter de deux opérations 
sémantico-cognitives, fondamentales et distinctes:

a) une opération de catégorisation, qui à partir d'un schéma cognitif primitif constitué de changements et de situations intermédiaires (niveau préconceptuel), subsume une portion de ce schéma primitif sous la détermination conceptuelle d'un procès (on parlera 'd'aspect conceptuel'),

b) une opération de monstration, i.e. de simulation de perception, qui donne à 'voir' tout ou partie du procès construit par l'opération précédente ('visée aspectuelle').

De façon très approximative, en français, la catégorisation correspond à l'aspect lexical (les lexèmes verbaux et leurs compléments dans lesquels le choix d'un grammème comme le déterminant du nom peut cependant jouer un rôle décisif), tandis que la monstration est indiquée par l'aspect grammatical (les conjugaisons et certaines périphrases, en relation avec divers marqueurs contextuels; cf. Gosselin, 2005: I29-I 53).

Cette double analyse présente des affinités évidentes à la fois avec la tradition aspectuelle guillaumienne, avec la perspective cognitive (voir en particulier le concept de 'fenêtrage', cf. Col et Victorri, 2007), mais aussi avec certains courants de sémantique formelle (voir d'une part Smith, I99I, Caudal et Vetters, 2006, et d'autre part Klein I994, Demirdache et Uribe-Etxebarria, 2002, Laca, 2005).

Le produit de ces deux opérations est représenté sous forme de structures d'intervalles disposées sur l'axe temporel. Alors que le moment d'énonciation est noté $[\mathrm{OI}, 02]$, l'opération de catégorisation produit un intervalle de procès $\left[\mathrm{B}_{1}, \mathrm{~B}_{2}\right]$, tandis que la monstration se traduit par l'ouverture d'une 'fenêtre', ou 'intervalle de référence' [I,II]. Le temps absolu se trouve défini par la relation entre l'intervalle de référence et celui de l'énonciation (conformément à la définition non standard du temps proposée par Reichenbach et argumentée par Klein, I994: 2 I-24). L'aspect résultant de la catégorisation ('aspect conceptuel') va correspondre à la nature des bornes du procès (bornes extrinsèques: procès atélique; bornes intrinsèques: procès télique, cf. Jackendoff I992) et à leur relation (procès ponctuel ou non). Quant à l'aspect produit par l'opération de monstration, ou 'visée aspectuelle', il se trouve représenté par la relation entre l'intervalle du procès $\left[\mathrm{B}_{\mathrm{I}}, \mathrm{B}_{2}\right]$ et l'intervalle de référence $[\mathrm{I}, \mathrm{II}]$, qui détermine ce qui est montré / perçu du procès. On est ainsi conduit à distinguer quatre visées aspectuelles de base en français, correspondant à quatre positions de la 'fenêtre de monstration' par rapport au procès. Ces quatre visées aspectuelles sont illustrées par la Figure 3 .

Remarquons que, contrairement à Vet (2002: I 84), il ne nous paraît pas exact de considérer que la visée inaccomplie (ou imperfective) porte nécessairement sur la phase médiane du procès, car il n'est pas impossible d'énoncer:

(I) (Que fais-tu?)

Je suis en train de manger. Je commence / je termine.

La visée inaccomplie indique uniquement que les bornes (et non les phases) initiale et finale ne sont pas prises en compte. Reconnaissons toutefois que Vet ne dit pas autre chose, simplement sa formulation découle du fait qu'il assimile la phase 
a) Visée aoristique (perfective, globale): le procès est montré dans son intégralité $(\mathrm{I}=\mathrm{B} 1, \mathrm{II}=\mathrm{B} 2)$; ex.: Il traversa le carrefour.

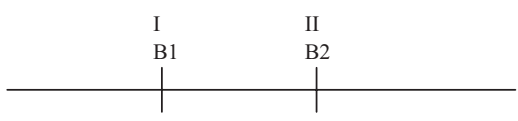

b) Visée inaccomplie (imperfective, sécante) : l'intervalle de référence est inclus dans celui du procès, dont les bornes initiale et finale ne sont pas prises en compte $(\mathrm{B} 1<\mathrm{I}, \mathrm{II}<\mathrm{B} 2)$; ex.: Il traversait le carrefour.

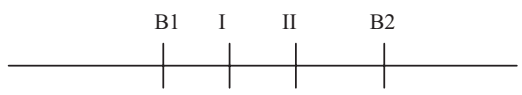

c) Visée accomplie: montre l'état résultant du procès $(\mathrm{B} 2<\mathrm{I})$; ex.: Il $a$ terminé son travail depuis dix minutes.

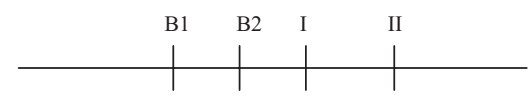

d) Visée prospective: présente la phase préparatoire (II < B1); ex.: Il allait traverser le carrefour.

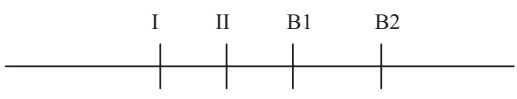

Figure 3. Les quatre visées aspectuelles de base du français.

initiale à la borne initiale, et la phase finale à la borne finale (voir ci-dessus $\S$ I). On peut donc dire que si les visées prospective et accomplie montrent respectivement les phases préparatoire et résultante du procès (constitutives de l'aspect externe), les visées aoristique et inaccomplie sélectionnent l'aspect interne, respectivement de façon globale (bornes comprises) et partielle (bornes exclues).

Dans ce cadre, la question de l'aspect de phase en français se laisse ainsi reformuler: les conjugaisons et les périphrases verbales permettent-elles de sélectionner les phases d'un procès par le biais d'une opération de catégorisation (aspect conceptuel), i.e. de construction d'un sous-procès, et/ou par la monstration (visée aspectuelle) de certaines parties du procès? (La question du statut lexical ou grammatical des marqueurs restant relativement secondaire et, en tout état de cause, pas totalement décidable).

On ne peut véritablement mesurer la portée de cette question que si l'on comprend à quel point les opérations de catégorisation et de monstration different entre elles: la catégorisation construit un (sous)procès, qui a un statut d'entité référentielle, qui possède un mode de déroulement spécifique et qui est décomposable en parties (phases). A l'inverse, la monstration ne produit aucune entité référentielle, mais une vue (visée) sur une entité préalablement construite (par la catégorisation). Cette visée est donc seconde par rapport au (sous)procès sur 
lequel elle porte, elle n'a évidemment pas de 'mode de déroulement' et n'est pas décomposable en parties.

\section{Coverbes de mouvement, de phase et de modalité D'ACTION VERSUS AUXILIAIRES DE VISÉE ASPECTUELLE}

Il se trouve que trois études récentes (Kronning, 2003, François, 2003, et Laca, 2005), issues d'horizons théoriques différents, et sans influences entre elles, convergent pour dissocier nettement, d'un point de vue syntaxique et distributionnel, deux classes de périphrases verbales en français. Or nous considérons que cette distinction syntaxique se laisse assez naturellement expliquer et interpréter en termes d'opérations sémantico-cognitives de catégorisation et de monstration; autrement dit, l'une des classes relève de l'aspect conceptuel (et déclenche donc la construction de sous-procès), tandis que l'autre marque la visée aspectuelle (en déterminant la position de la 'fenêtre' par rapport au procès).

Cette analyse nous conduit à proposer une typologie des coverbes (représentée par la Figure 4) que nous allons essayer de justifier.

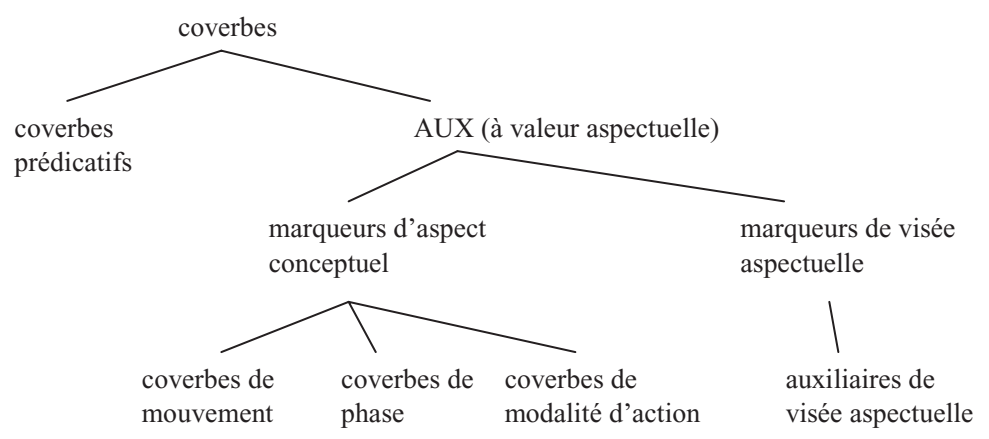

Figure 4. typologie des coverbes.

Nous considérons comme coverbe, à la suite Kronning (2003: 232), 'tout verbe qui se construit avec un mode impersonnel', i.e. tout verbe qui peut être suivi d'un infinitif ou d'un participe. Parmi les coverbes, on isole ensuite la classe des auxiliaires au sens large (AUX) qui ne recouvre que les coverbes qui excluent la construction '(ce) que p'. Ce critère est discuté et adopté entre autres par Gross (I999: 8), Borillo (2005: 7I) et Lamiroy (I999: 38), qui l'étend à l'ensemble des langues romanes:

'La propriété formelle commune qui distingue les auxiliaires romans du reste des verbes est qu'ils se font suivre d'un infinitif tout en excluant la complétive Que P. Ce dernier trait est en même temps leur propriété syntaxique définitoire.'

Il conduit, par exemple, à écarter des verbes volitifs comme vouloir, souhaiter, décider de la classe des AUX, dans la mesure où ils acceptent d'être suivis de la complétive conjuguée, tandis que seront retenus des coverbes de mouvement comme partir, 
courir, rentrer de, sortir de, parce qu'ils acceptent l'infinitif (ex: 'courir faire les courses'), mais excluent la construction en '(ce) que p'. Remarquons que ces coverbes de mouvement étaient traditionnellement tenus pour des verbes 'pleins' (prédicatifs) suivis, dans ces tours, d'une subordonnée circonstancielle de but, avec ellipse de la préposition pour. Cette analyse a été très précisément réfutée par Lamiroy (I983), qui montre que ces constructions présentent un ensemble de caractéristiques et de contraintes spécifiques. Certains auteurs, comme Gross et Prandi (2004: I26I 3 I) considèrent cependant toujours qu'il s'agit de verbes pleins, mais suivis d'une subordonnée de destination-but à valeur d'argument (et non de circonstant). Or cette analyse (qu'ils appliquent aussi à un verbe comme 's'efforcer de'), outre qu'elle ne permet pas de rendre compte des contraintes très strictes qui pèsent sur ces constructions, se heurte - comme l'a montré Vet (1987) - à des exemples du type:

(2) Je cours chez le boucher acheter un rôti de veau

où deux expressions référentielles distinctes remplissent un même rôle de destination-but. Pour éviter une violation manifeste du 'critère thématique' des théories chomskyennes (Chomsky, I98 I, Pollock, I997: 6I) ou du 'principe de non redondance fonctionnelle' de Milner (I982: I40-I79), il faudrait alors supposer (avec Gross et Prandi, 2004: I27) que les verbes de mouvement régissent deux places argumentales correspondant à des compléments essentiels (de destination et de but) qui ne seraient, paradoxalement, réalisés tous les deux que de façon exceptionnelle (comme dans l'exemple 2). Nous préférons adopter la position de Lamiroy (I983: i I6), qui voit dans ces verbes 'un cas intermédiaire entre les verbes pleins régissant un complément à l'infinitif et les auxiliaires d'aspect'. Cette analyse s'inspire explicitement de celle de Damourette et Pichon (I9I I-I936, III, §Io55) qui reprennent le concept freudien de 'progrédience' pour décrire le fait que 'dans Louis vient déjeuner (...) l'action de déjeuner est en quelque sorte déjà entamée par les pas que fait Louis vers la maison où il doit manger'. En d'autres termes, le coverbe de mouvement venir exprime ici le mode de déroulement de la phase préparatoire du procès dénoté par déjeuner. Il contribue donc à l'expression de l'aspect de phase.

Prenons garde cependant aux verbes polysémiques, dont certains emplois peuvent relever d'un mode de fonctionnement, tandis que d'autres appartiennent à une autre classe. C'est, par exemple, le cas de 's'apprêter à', qui, à première vue, parait accepter aussi bien l'infinitif ('s'apprêter à partir') que la forme conjuguée au subjonctif ('s'apprêter à ce que les prix augmentent'). Pourtant, à la différence de Borillo (2005: 76), nous ne refuserons pas le statut d'AUX à 's'apprêter à' en général, car il ne présente pas les mêmes caractéristiques syntactico-sémantiques dans les deux constructions. Suivi du subjonctif, il devient quasi-synonyme de 's'attendre à ce que', et prend une valeur statique (il est incompatible avec 'être en train de'; ex. $3 \mathrm{a}$ ), tandis que lorsqu'il est suivi de l'infinitif, il signifie 'faire ses préparatifs en vue de' (il est alors dynamique; ex. 3):

(3a) ?*Il est en train de s'apprêter à ce que les prix augmentent

(3b) Il est en train de s'apprêter à partir. 
Nous considérerons donc que seul le premier emploi est exclu de la classe des AUX. Autrement dit, le test de la substitution de la forme conjuguée à la forme infinitive ne vaut que s'il n'entraîne pas de changement de sens.

La classe des AUX (dont on ne retient ici que ceux qui ont une valeur aspectuelle, au sens où ils permettent de sélectionner des phases de procès) se divise à son tour en deux sous-classes: celle qui recouvre les coverbes de mouvement, de modalité d'action ('s'efforcer de') et de phase ('commencer à), et celle des auxiliaires de visée aspectuelle. Ce sont précisément les travaux évoqués, de Kronning (2003), François (2003) et Laca (2005) qui nous conduisent à opérer cette partition (déjà évoquée par François, I993: 53 et 67 n.5). Car ils mettent en évidence, au moyen de tests syntaxiques distincts mais convergents, l'existence de deux classes distributionnelles parmi les AUX d'aspect. Or comme on l'a annoncé, ces différences de fonctionnement s'expliquent assez naturellement si l'on admet que les coverbes de mouvement, de modalité d'action, et de phase (désormais 'coV-MAP') marquent la catégorisation d'une phase, i.e. la construction d'un sous-procès, tandis que les auxiliaires précisent la visée aspectuelle ('aux-VA'), la monstration de tout ou partie d'un procès ou d'un sous procès.

On comprend ainsi pourquoi les aux-VA peuvent porter sur les coV-MAP, alors que la réciproque est fausse (cf. François, 2003: ch. 5, Laca, 2005; signalons toutefois que ni François ni Laca ne prennent en compte les coverbes de mouvement).

\begin{tabular}{|l|l|l|}
\hline venir de & $\begin{array}{l}\text { cesser de } \\
\text { être sur le point de } \\
\text { être en train de }\end{array}$ & Vinf \\
& $\begin{array}{l}\text { commencer à } \\
\text { s'apprêter à } \\
\text { partir } \\
\text { rentrer de }\end{array}$ & \\
& & \\
& & \\
\hline
\end{tabular}

Figure 5. aux-VA portant sur des coV-MAP.

\begin{tabular}{|l|l|l|}
\hline *commencer à & $\begin{array}{l}\text { être sur le point de } \\
\text { être en train de }\end{array}$ & Vinf \\
*inir de & venir de & \\
*cesser de & & \\
*s'apprêter à $^{*}$ aartir & & \\
*rentrer de & & \\
\hline
\end{tabular}

Figure 6.coV-MAP ne pouvant porter sur des aux-VA.

Il est en effet concevable, selon les principes adoptés ci-dessus, que la monstration porte sur une sous-catégorie de procès, mais en aucune façon que la catégorisation puisse affecter la monstration (la catégorisation devant toujours précéder conceptuellement la monstration). 
De même, Laca (2005) montre que seuls les coV-MAP sont récursifs. Il existe certes des contraintes, fortes et complexes, sur leurs combinaisons, mais on peut dire que pour tout coV-MAP, il existe au moins une sous-classe de coV-MAP susceptibles de le prendre dans leur portée, alors qu'un aux-VA ne peut jamais être dans la portée d'un autre.

\begin{tabular}{|c|c|c|}
\hline $\begin{array}{l}\text { cesser de } \\
\text { commencer à } \\
\text { continuer de }\end{array}$ & $\begin{array}{l}\text { s'apprêter à } \\
\text { hésiter à }\end{array}$ & Vinf \\
\hline $\begin{array}{l}\text { s'apprêter à } \\
\text { hésiter à }\end{array}$ & $\begin{array}{l}\text { cesser de } \\
\text { commencer à } \\
\text { continuer de }\end{array}$ & Vinf \\
\hline $\begin{array}{l}\text { courir } \\
\text { s'efforcer de }\end{array}$ & $\begin{array}{l}\text { s'installer à } \\
\text { se préparer à }\end{array}$ & Vinf \\
\hline
\end{tabular}

\begin{tabular}{|c|c|c|}
\hline $\begin{array}{l}\text { tarder à } \\
\text { se hâter de }\end{array}$ & $\begin{array}{l}\text { rentrer de } \\
\text { partir } \\
\text { finir de }\end{array}$ & Vinf \\
\hline $\begin{array}{l}\text { se précipiter à } \\
\text { rentrer de } \\
\text { partir }\end{array}$ & finir de & Vinf \\
\hline
\end{tabular}

Figure 7.coV-MAP portant sur des coV-MAP.

\begin{tabular}{|l|l|l|}
\hline *venir de & \multicolumn{1}{|c|}{$\begin{array}{l}\text { ettre sur le point de } \\
\text { etre en train de }\end{array}$} & Vinf \\
\hline *être sur le point de & $\begin{array}{l}\text { venir de } \\
\text { etre en train de }\end{array}$ & Vinf \\
\hline
\end{tabular}

Figure 8. aux-VA ne pouvant porter sur des aux-VA.

Il est de fait envisageable de découper un sous-procès à l'intérieur d'un autre sous-procès, alors qu'on ne peut même concevoir ce que serait une visée aspectuelle portant sur une autre visée. Remarquons que si, à première vue, 'il va être en train de Vinf constitue un contre-exemple, on peut cependant montrer que, dans ce cas, aller n'est pas un auxiliaire de visée aspectuelle, mais un auxiliaire à valeur temporelle, cf. ci-dessous.

Par ailleurs, Kronning (2003: 235-239) observe, dans le cadre de l'analyse stratificationnelle de la phrase, que seuls les coV-MAP, à la différence des auxVA, sont rhématiques au sens où ils sont focalisables par focalisation simple (sans marquage intonatif particulier). Cette propriété conduit à prédire que seuls les coV-MAP pourront apparaitre naturellement en fin d'énoncé, à la suite soit d'une pronominalisation, soit d'une ellipse de l'infinitif.

Remarquons que les tours 'il est sur le point' et 'il est en train' sont tout de même possibles, mais moyennant des contextes contrastifs et/ou une accentuation particulière, comme dans cet exemple:

(4) 'Hé quoi! Elles sont parties depuis longtemps! Parties ou sur le point!' (Kleist: Le Prince de Hombourg, I, 4, trad. R. Orthmann et E. Recoing, Actes Sud). 


\begin{tabular}{|l|}
\hline Il commence \\
continue \\
finit \\
cesse \\
hésite \\
s'y met \\
s'y acharne \\
s'y apprête \\
y court \\
s'y précipite \\
en revient
\end{tabular}

Figure 9.coV-MAP focalisés par focalisation simple.

$$
\begin{aligned}
& \text { ?*Il(en) est sur le point } \\
& \text { (en) est en train } \\
& \text { (en) est en passe } \\
& { }^{\#} I l \quad y v a \\
& \text { en vient }
\end{aligned}
$$

Figure Io. aux-VA non focalisables par focalisation simple.

Dans la mesure où il ne s'agit pas là de focalisation simple, mais de focalisation spécialisée (au sens de Nølke 200I: I37) cela ne met pas en cause le caractère non rhématique de ces éléments. Quant aux constructions ' $i l y v$ ' et 'il en vient', elles impliquent toutes deux qu'il y ait déplacement du sujet (et donc coverbe de mouvement). Quand ce déplacement est exclu la pronominalisation est impossible:

(5a) il va pleuvoir $\longrightarrow *$ il y va

(5b) il vient de pleuvoir $\longrightarrow *$ il en vient.

C'est pourquoi, on distinguera, au moins, deux types d'emplois d'aller et venir de lorsqu'ils sont suivis de l'infinitif: un emploi de coverbe de mouvement, rhématique, et un emploi non rhématique d'auxiliaire de visée aspectuelle (voir ci-dessous).

Selon l'analyse de Kronning (2003: 235), 'la stratification énonciative de la phrase (...) est la restructuration de la phrase, lors de l'énonciation, en "substrat" et "foyer" (on trouve une analyse comparable dans Lambrecht, 2004: 33 sq.). Or le rhème est le 'domaine de la focalisation stratificationnelle', c'est-à-dire que c'est le domaine dans lequel l'énonciation va pouvoir sélectionner (focaliser) une valeur référentielle susceptible de saturer une variable comprise dans le substrat. On comprend dès lors comment notre hypothèse d'une correspondance entre d'une part coV-MAP et catégorisation (constitution de sous-procès), et d'autre part aux-VA et monstration, rend naturellement compte de cette opposition syntaxique: seul un sous-procès, 
par opposition à la visée aspectuelle, a le statut référentiel nécessaire pour pouvoir être focalisé, et venir saturer la variable du substrat.

Prenant appui sur Damourette et Pichon (I9I I-I940, V, § I605), Kronning (2003: 24I-242) montre enfin que seuls les coverbes non auxiliaires (et donc les coV-MAP) tolèrent des compléments adverbiaux 'de manière'.

Il commence avec précaution à Vinf
continue
s'apprête

Il rentre précipitamment (de/à) Vinf part s'acharne

Figure I I.coV-MAP avec complément de manière.

$$
\begin{aligned}
& \text { *Il est en train avec précaution de Vinf } \\
& \text { est sur le point } \\
& \text { \#Il vient avec précaution (de) Vinf } \\
& \text { va }
\end{aligned}
$$

Figure I2. aux-VA incompatibles avec des compléments de manière.

Observons, là encore, qu'aller et venir n'acceptent ces compléments que s'ils indiquent un mouvement physique (dans leur emploi de coverbe de mouvement):

(6a) Il va précipitamment acheter son journal

(6b) *Il va précipitamment pleuvoir.

Ce contraste syntaxique entre coV-MAP et aux-VA s'explique aisément selon notre hypothèse: seuls les sous-procès (construits par les coV-MAP), à la différence des visées aspectuelles, ont des modes de déroulement spécifiques, exprimables au moyen de compléments de manière.

L'application systématique de ces différents critères aux AUX à valeur aspectuelle conduit au classement représenté par la Figure I3.

Ce classement appelle plusieurs remarques:

a) La distinction entre coverbes de phase, de modalité d'action et de mouvement n'est pas de nature proprement aspectuelle: les premiers n'indiquent que la phase concernée, les autres précisent de surcroît son mode de déroulement (sur les coverbes de modalité d'action, voir François 2003, chap. 5).

b) Un même élément peut présenter divers emplois en contexte, qui relèvent de différentes sous-classes. Prenons par exemple les verbes aller et venir, qui ont suscité une littérature très abondante (voir en particulier, sur le futur périphrastique, Vet I993 et 200I). Il apparaît que ces deux verbes peuvent fonctionner, entre autres, comme:

I) verbes pleins (ex.: aller à / venir de Marseille),

2) coverbes de mouvement (ex.: aller / venir (en voiture) (de) faire les courses), 


\begin{tabular}{|c|c|c|}
\hline \multicolumn{3}{|c|}{$\begin{array}{l}\text { auxiliaires de visée aspectuelle: } \\
\text { visée prospective (sur phase préparatoire): }\end{array}$} \\
\hline \multicolumn{3}{|c|}{$\begin{array}{r}\text { visée inaccomplie (sur aspect interne, bornes exclues): } \begin{array}{l}\text { etre en train de } \\
\text { (en) être à }\end{array}\end{array}$} \\
\hline \multicolumn{3}{|c|}{$\begin{array}{ll}\text { visée accomplie (sur phase résultante): } & \text { etre/avoir }+ \text { PPé } \\
& \text { venir de }\end{array}$} \\
\hline coverbes de phase & $\begin{array}{l}\text { coverbes de modalité } \\
\text { d'action }\end{array}$ & coverbes de mouvement \\
\hline $\begin{array}{l}\text { phase préparatoire: } \\
\text { être prêt à } \\
\text { s'apprêter à } \\
\text { se préparer à }\end{array}$ & $\begin{array}{l}\text { se proposer de } \\
\text { hésiter à } \\
\text { tarder à }\end{array}$ & $\begin{array}{ll}\text { partir } & \text { s'installer à } \\
\text { aller } & \text { s'arrêter à } \\
\text { monter } & \\
\text { courir } & \end{array}$ \\
\hline $\begin{array}{l}\text { phase initiale: } \\
\text { commencer à } \\
\text { se mettre à }\end{array}$ & \multirow{3}{*}{$\begin{array}{l}\text { s'acharner à } \\
\text { s'efforcer de } \\
\text { se dépêcher de } \\
\text { mettre du temps à } \\
\text { être long à }\end{array}$} & \\
\hline $\begin{array}{l}\text { phase médiane: } \\
\text { continuer de } \\
\text { être occupé à } \\
\text { persévérer à }\end{array}$ & & \\
\hline $\begin{array}{l}\text { phase finale: } \\
\text { cesser de } \\
\left(s^{\prime}\right) \text { arrêter de } \\
\text { finir de }\end{array}$ & & \\
\hline phase résultante: & & $\begin{array}{l}\text { (re)venir de } \\
\text { rentrer de } \\
\text { (re)descendre de } \\
\text { sortir de }\end{array}$ \\
\hline
\end{tabular}

Figure I3. classement des AUX à valeur aspectuelle. 
3) auxiliaires de temps à valeur de futur ('Dans cinq minutes, on va bien rigoler', exemple attesté cité par Sundell, I99I et commenté par Vet, I993: 79) ou de passé (ex.: 'Nous venons récemment de perdre encore une sœur charmante', Lamartine, cité par Gougenheim, I929: I27),

4) auxiliaires de visée aspectuelle prospective ('Je vois que Pierre va se fâcher', Vet, I993: 74) ou accomplie ('Chatterton venait d'expirer depuis peu de jours lorsque parurent à la fois un poème burlesque et un pamphlet sur sa mort', Vigny cité par Gougenheim, I929: I27; le circonstanciel [depuis + durée] indique précisément ce type de visée aspectuelle, cf. Gosselin I996: 27).

c) Les coV-MAP peuvent marquer:

I) la coïncidence du sous-procès avec une phase du procès (ex.: commencer à, continuer à, finir de);

2) l'inclusion du sous-procès dans une phase (ex.: s'apprêter à, hésiter à ...); c'est ainsi que rentrer de dans 'rentrer de faire les courses' marque l'inclusion (et pas nécessairement la coïncidence) du sous-procès dans la phase résultante, si bien qu'une visée prospective sur ce sous-procès ne conduit pas nécessairement à la phase précédente: de (7), on ne peut inférer (8).

(7) Paul était sur le point de rentrer de faire ses courses

(8) Paul était encore en train de (finir de) faire ses courses.

Car il se peut très bien qu'en (7) Paul se trouve déjà dans la phase résultante (i.e. qu'il ait fini les courses, mais n'ait pas encore commencé à rentrer);

3) le recouvrement, éventuellement partiel, de plusieurs phases (ex.: se hâter de, s'acharner à, s'efforcer de...).

d) Les coverbes de modalité d'action portant sur l'aspect interne (ex.: s'acharner à, se hâter de, se dépêcher de, être long à ....) sont conduits à exprimer la phase préparatoire du procès si ce dernier est ponctuel (i.e. si sa phase interne n'est pas accessible). Gosselin (I996: I72-I76) montre que ce mode résolution de conflit par glissement vers la phase préparatoire est régulier et prédictible. Cela explique, entre autres, pourquoi 'se dépêcher de' devient équivalent de 'ne pas perdre de temps avant de' lorsque le procès est ponctuel:

(9) Il s'est dépêché de sortir ( $\approx$ il n'a pas perdu de temps avant de sortir).

Nous allons voir maintenant comment représenter et interpréter ces phénomènes dans le cadre du modèle SdT.

\section{VISÉES ASPECTUELLES ET SOUS-PROCÈS DANS LE MODÈLE SDT}

Les sous-procès, construits sur la base des instructions codées par les coV-MAP vont être représentés par des intervalles notés $\left[\mathrm{B}_{1}^{\prime} \mathrm{B}_{2}^{\prime}\right],\left[\mathrm{B}^{\prime \prime} \mathrm{I}_{1} \mathrm{~B}^{\prime \prime} 2\right]$, etc. Par souci de lisibilité, nous dupliquons les axes temporels (en fait, il ne s'agit ici que de représentations iconiques de structures qui doivent être conçues comme des réseaux de contraintes sur des variables d'intervalles). La Figure I4 en illustre un exemple: 


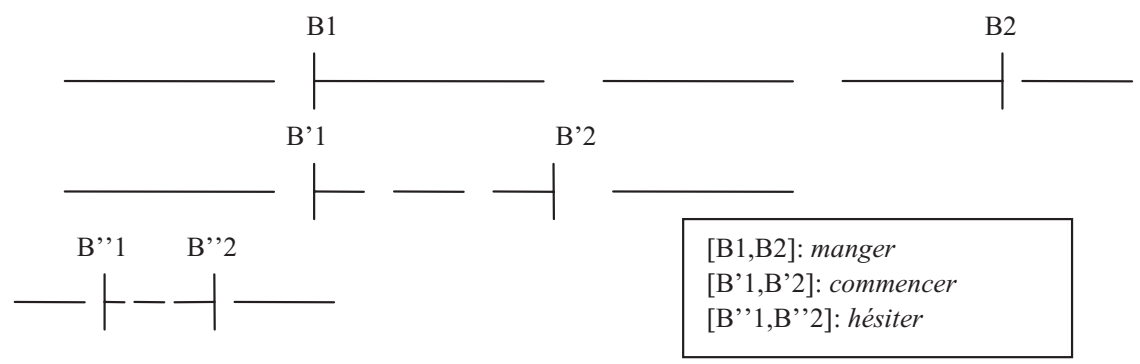

Figure I4. représentation iconique de 'hésiter à commencer à manger'.

Le sous-procès marqué par commencer à coïncide avec la phase initiale du procès manger, tandis que celui qu'exprime hésiter à est inclus dans la phase préparatoire du sous-procès, laquelle correspond également à la phase préparatoire du procès manger. Cette structure rend donc compte à la fois du fait que 'hésiter à commencer à manger' est quasi-synonyme de 'hésiter à manger', et de ce que 'avoir hésité à commencer à manger' n'implique pas 'commencer à manger', dans la mesure où $\mathrm{B}^{\prime \prime} 2$ ne coïncide pas nécessairement avec $\mathrm{B}^{\prime} \mathrm{I}$ (la phase résultante du sous-procès hésiter peut commencer avant le début du sous-procès commencer, lequel coïncide avec celui du procès manger).

Quant à la visée aspectuelle, marquée par l'interaction de divers éléments dont la conjugaison et d'éventuels aux-VA, elle se traduit par l'ouverture d'une 'fenêtre de monstration', indiquée par l'intervalle de référence ([I,II]), qui se positionne à la fois vis-à-vis du sous-procès le plus enchâssé (i.e. celui qui correspond au stade le plus élevé de décomposition des (sous-)procès), et relativement aux autres procès et sous-procès éventuels de la structure. On parlera de visée aspectuelle directe dans le premier cas, et de visée indirecte dans le second. Si l'on reprend les quatre types de visée aspectuelle présentés au $\S 3$, on constate que le type de visée peut être différent pour les divers (sous-)procès d'une même construction. Ainsi, dans l'énoncé

(Io) 'Au château d'Edimbourg, John Benstede, clerc et émissaire spécial d'Edouard d'Angleterre, s'apprêtait également à conclure sa mission.' (P.C. Doherty, La couronne dans les ténèbres, I0/I 8, éd., I996: 21 I)

la visée directe, marquée par l'imparfait, est inaccomplie sur la phase préparatoire catégorisée par s'apprêter $\left(\mathrm{B}^{\prime}{ }_{\mathrm{I}}<\mathrm{I}<\mathrm{II}<\mathrm{B}^{\prime} 2\right.$ ), mais prospective sur le procès (II $<$ $\mathrm{B}_{\mathrm{I}}$ ), comme le montre la Figure i 5 .

Soit un autre exemple, construit:

(I I) (Quand je l'ai rencontré) il venait de commencer à écrire son chapitre

A vrai dire, la Figure I6 n'illustre qu'une des interprétations possibles de l'exemple (I I), car 'venir de' ne contraint pas directement la position de l'intervalle de référence relativement au procès 'écrire son chapitre': on pourrait enchaîner par 'et il l'avait déjà (presque) terminé'. 


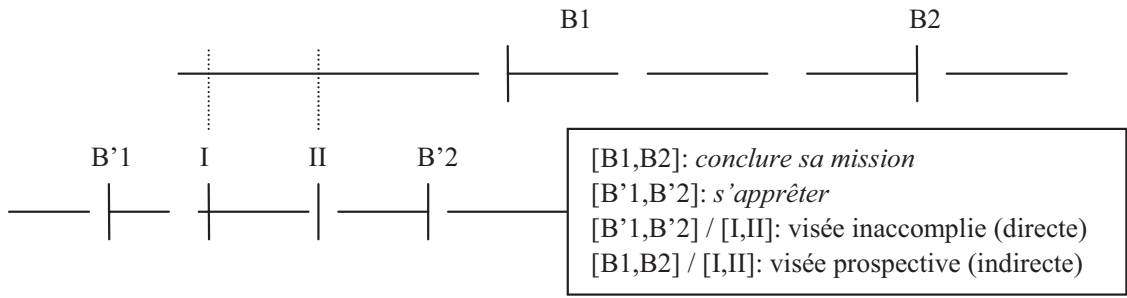

Figure I 5. représentation iconique de '(il) s'apprêtait à conclure sa mission'.

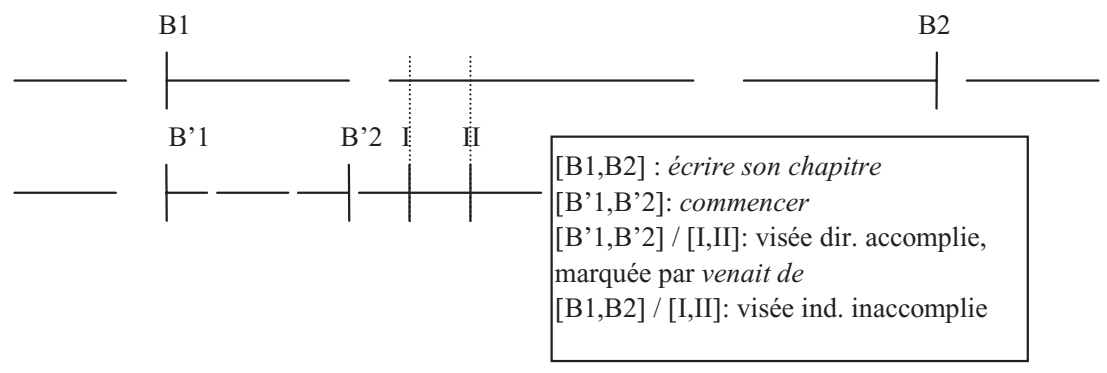

Figure I6. représentation iconique de 'il venait de commencer à écrire son chapitre'.

\section{INTERPRÉtATion temporelle et Modale Des STRUCTURES D'INTERVALLES}

Le temps absolu étant défini par la relation entre l'intervalle de référence ([I,II]) et l'intervalle d'énonciation ([O I,02]), il suit que, dans des exemples comme (IO) et (I I), on comprend, à cause de l'instruction temporelle associée à l'imparfait, que l'intervalle de référence est antérieur au moment de l'énonciation (II < OI), mais aucune contrainte ne pèse sur la position des diverses bornes situées à droite de II relativement à [OI,O2] (c'est pourquoi nous avons préféré ne pas faire figurer cet intervalle d'énonciation dans les représentations iconiques). Cela revient à dire, par exemple, que dans l'exemple (II), le fait de finir d'écrire son chapitre peut aussi bien être envisagé dans le passé que dans le présent ou l'avenir.

Par ailleurs, Gosselin (2005: 88-96) a montré qu'à l'indicatif, la borne finale de l'intervalle de référence (II) constituait une 'coupure modale' entre l'irrévocable (à gauche) et le possible (à droite). C'est pourquoi tout ce qui, dans ces exemples, est situé à droite de II est simplement possible, au sens où tout procès ou partie de procès qui se trouve à droite de II peut ne pas se réaliser. C'est ainsi que, dans le roman d'où est extrait l'exemple io, le personnage est assassiné avant de pouvoir conclure sa mission.

Ces caractéristiques temporelles et modales des structures d'intervalles sont évidemment essentielles dans une perspective d'analyse sémantique automatique et d'extraction d'information, car elles permettent de prédire certaines des inférences 
que l'on peut légitimement tirer de l'énoncé. En effet, contrairement à ce qu'affirment Reyle, Rossdeutscher et Kamp (2007: 630), la visée aspectuelle, bien que n'ayant pas de statut référentiel, joue un rôle décisif au plan vériconditionnel. Comparons, à cet égard, les exemples:

(I2a) Il descendit faire les courses

(I $\mathrm{b}$ ) Il allait descendre faire les courses

(I2c) Il était en train de descendre faire les courses

(I2d) Il venait de descendre faire les courses.

La structure associée à (I2a) correspond à la Figure I7.

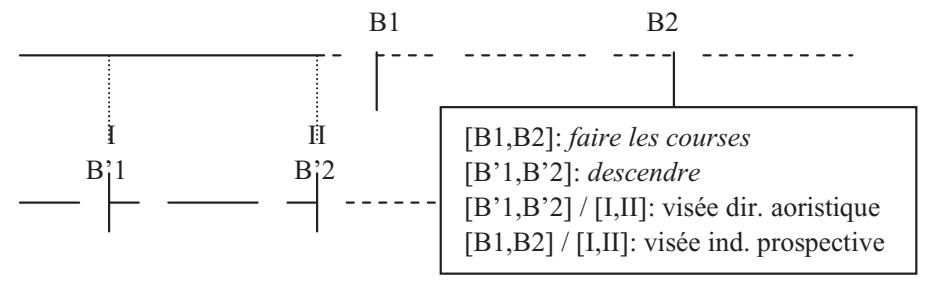

Figure I7. représentation iconique de 'il descendit faire les courses'.

On comprend que le sous-procès descendre est réalisé dans sa totalité, mais que le procès 'faire les courses', vu de façon prospective, reste dans le domaine du possible, comme le montre le fait que l'on puisse enchaîner sur

(I3) quand il prit conscience qu'il n'avait plus d'argent qui annule uniquement le procès faire les courses (et non le sous-procès).

Les exemples (I 2 b-d) ne different de (I 2a) que par la visée aspectuelle et donc par la position de l'intervalle de référence $([\mathrm{I}, \mathrm{II}])$. Or les inférences qu'on peut en tirer sont très différentes. En ( $\mathrm{I} 2 \mathrm{~b}$ ) le sous-procès lui-même, présenté prospectivement, est simplement possible, de sorte que l'enchainement (I3) annule les deux (sous-)procès.

En revanche, (I2c) illustre le 'paradoxe imperfectif, appliqué au sous-procès descendre, dont le début relève de l'irrévocable, et la fin du possible: l'enchaînement (I3) ne peut annuler le début du sous-procès.

L'exemple (I2d) est virtuellement ambigu. La visée accomplie, marquée par 'venir $d e$, situe l'intégralité du sous-procès dans l'irrévocable (il n'est plus annulable). Mais la position de l'intervalle de référence relativement au procès 'faire les courses' n'est pas contrainte. Si bien que l'on pourrait accepter, à la suite de (I 2d), les enchaînements suivants:

(I 4a) Il n'avait pas commencé ses achats quand il prit conscience qu'il n'avait plus d'argent

(I 4b) Il avait à peine commencé ses achats qu'il prit conscience que ...

(I4c) Il avait à peine terminé ses achats qu'il prit conscience que ... 


\section{LA QUESTION DE LA RÉCURSIVITÉ}

C'est, selon Laca (2004 et 2005), la propriété de récursivité qui distingue fondamentalement ce que nous appelons coV-MAP et aux-VA, car seuls les premiers sont récursifs. La valeur sémantique qu'elle confere à ces deux classes d'AUX est comparable à la nôtre (elle s'inspire explicitement des modèles de Smith, I99 I et de Klein, I994). Mais son traitement de la récursivité est tout différent. Dans le cadre chomskyen de l'analyse de la temporalité, défini par Demirdache et Uribe Etxebarria (2002), elle propose de traiter la récursivité des coverbes au moyen d'une règle syntagmatique, illustrée par la Figure i 8.

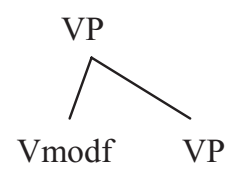

Figure I8. récursivité du VP (le verbe modificateur, Vmodf, correspond au coverbe non auxiliaire).

En revanche, elle considère que la visée aspectuelle correspond à un nœud ASP$\mathrm{P}$, qui n'est pas récursif. Or, comme, dans ce cadre théorique, on représente par un même dispositif des relations sémantiques et les marqueurs qui les expriment, ces hypothèses deviennent lourdes de conséquences difficiles à défendre. Ainsi la non-récursivité du nœud ASP-P oblige à admettre que les temps simples comme le présent, le futur ou même l'imparfait sont dépourvus de valeur aspectuelle, sans quoi cette non-récursivité serait prise en défaut dans un tour comme 'il venait de manger', dans lequel l'aspect serait doublement marqué par l'auxiliaire et par l'imparfait. Et s'il l'on envisage la possibilité d'énoncer 'dès qu'ils furent / on été / avaient été en train de manger'(cf. Gosselin, I996: 250-25I), ou 'ils furent / ont été / avaient été sur le point de partir', il apparaît nécessaire d'étendre encore cette analyse au passé simple et aux temps composés, c'est-à-dire à l'ensemble des conjugaisons du français (curieusement, Laca, 2004 et 2005 considère que 'être en train de' est incompatible avec le passé simple, le passé composé, ainsi qu'avec le passif; par ailleurs, elle ne classe pas 'être sur le point de' parmi les auxiliaires de visée aspectuelle, mais dans les modificateurs d'éventualité).

La conception mise en œuvre dans le modèle SdT des relations entre marqueurs (lexèmes, grammèmes et constructions syntaxiques) et valeurs sémantiques est toute différente. On considère en effet que les valeurs temporelles, aspectuelles et modales résultent le plus souvent d'interactions complexes entre divers marqueurs, ainsi que de la prise en compte de contraintes pragmatico-référentielles. On admet, par principe, que la visée aspectuelle n'est pas récursive, dans la mesure où il n'est simplement pas concevable qu'une visée porte sur une autre visée. En revanche, il se peut très bien qu'une visée soit construite à partir d'instructions codées par des marqueurs différents (aux-VA, flexions verbales, circonstanciels de durée ...). On se limitera ici à observer qu'il est cependant impossible de combiner, pour l'expression d'une même visée aspectuelle, deux marqueurs de visée aspectuelle du 
même type morpho-syntaxique (deux aux-VA, deux flexions verbales, ou deux circonstanciels de durée). Les relations de compatibilité entre les différents aux-VA et les conjugaisons demanderaient un examen approfondi.

Quant à la récursivité des coV-MAP, elle repose sur une propriété singulière des procès envisagés du point de vue de leur structure phasale: toute phase de procès, dès lors qu'elle est catégorisée comme sous-procès, peut à son tour être décomposée en phases, selon la même structure méréotopologique, représentée par la Figure I. On aura reconnu là la propriété d'autosimilitude, caractéristique des objets fractals: la partie et le tout, de même que la partie de la partie, possèdent une structure identique. Et l'on pourrait aller plus loin en observant que s'il est possible de construire des macroprocès, entre autres par l'itération d'un même procès modèle, ces macroprocès exhibent encore la même structure méréotopologique, et leurs phases sont également catégorisables au moyen des coverbes que nous avons décrits:

(I 5) Il hésitait à/commença à/continuait de/cessa de prendre sa leçon de piano le lundi après-midi.

Comme il est encore possible d'itérer des phases:

(I6) A chaque leçon, il se mettait très rapidement à jouer

et donc de construire des phases d'itérations de phases:

(I7) Il continuait de se mettre très rapidement à jouer, à chaque leçon.

On finit par obtenir des structures d'une grande complexité, mais qui reposent toujours sur une combinatoire d'occurrences de la même structure phasale.

\section{CONCLUSION}

L'aspect de phase repose sur une même structure phasale (comprenant deux phases externes et trois phases internes), que l'on retrouve, au niveau de l'aspect conceptuel, aussi bien dans les procès que dans les parties de procès (propriété d'autosimilitude), et sur l'existence de deux opérations sémantico-cognitives complémentaires qui permettent, chacune à sa façon, de rendre les phases saillantes:

a) une opération de catégorisation qui construit des sous-procès (lesquels sont coïncidents, inclus, ou englobants vis-à-vis des phases),

b) une opération de monstration qui donne à voir tout ou partie du procès ou du sous-procès (visée aspectuelle).

En français, les conjugaisons indiquent (en relation avec d'autres marqueurs) la visée aspectuelle, tandis que les périphrases verbales se laissent répartir en deux classes sur la base de critères syntaxiques et distributionnels: celles qui mettent en œuvre des coverbes déclenchant la construction (par catégorisation) de sous-procès, et celles qui, au moyen d'auxiliaires de visée aspectuelle, permettent la monstration de certaines phases de procès ou de sous-procès.

En somme, alors que des études ont montré l'impossibilité de distinguer nettement deux classes de périphrases verbales sur la seule base de l'opposition entre 
lexique et grammaire, une telle distinction s'avère néanmoins possible et pertinente si l'on prend appui sur les opérations sémantico-cognitives fondamentales codées par ces périphrases. Il en résulte un nouveau classement des auxiliaires (au sens large) à valeur aspectuelle (i.e. permettant de sélectionner des phases), qui oppose, d'une part, les coverbes de mouvement, de modalité d'action et de phase (qui construisent des sous-procès), et d'autre part, des auxiliaires de visée aspectuelle (qui servent à situer la 'fenêtre de monstration' relativement au (sous-)procès).

Intégrée au modèle SdT, cette analyse permet enfin de calculer les inférences que l'on peut tirer des énoncés, à propos de la réalisation des procès et sous-procès présentés. On montre ainsi que, bien que dépourvue de statut référentiel, la visée aspectuelle n'en est pas moins déterminante pour l'interprétation vériconditionnelle des énoncés.

Adresse pour correspondance:

Laurent Gosselin

Université de Rouen

7 rue Thomas Becket

76821 Mont-Saint-Aignan cedex

France

e-mail: Laurent.Gosselin@univ-rouen.fr

\section{RÉFÉRENCES}

Arrivé, M., Gadet, F. et Galmiche, M. (1986). La grammaire d'aujourd'hui. Paris: Flammarion.

Barcelo, G. J. et Bres, J. (2006). Les Temps de l'indicatif en français. Paris: Ophrys.

Blanche-Benveniste, Cl. (200I). Auxiliaires et degrés de 'verbalité'. Syntaxe E Sémantique, 3: 75-98.

Borillo, A. (1986). La quantification temporelle: durée et itérativité en français. Cahiers de grammaire, I I: I I7-I 56.

Borillo, A. (2005). Peut-on identifier et caractériser les formes lexicales de l'aspect en français? Dans: H. Bat-Zeev Shyldkrot et N. Le Querler (dir.), Les périphrases verbales. Amsterdam, Benjamins, pp. 67-82.

Borillo, A. (2006). Quelques formes lexicales de l'aspect adnominal en français. Cahiers de grammaire, 30: $2 \mathrm{I}-38$.

Brunot, F. (1922). La Pensée et la langue. Paris: Masson.

Caudal, P. et Vetters, C. (2006). Les temps verbaux: des connecteurs temporels qui s'ignorent? Cahiers Chronos, I5: I05-137.

Cazati, R. et Varzi, A. (1999). Parts and Places. The Structure of Spatial Representation. Cambridge, MA: MIT Press.

Chomsky, N. (198I). Lectures on Government and Binding, Amsterdam: Foris.

Col, G. et Victorri, B. (2007). Comment formaliser en linguistique cognitive? Opération de fenêtrage et calcul du sens temporel. Dans: G. Achard-Bayle et M.-A. Paveau (dir.), Cognition, discours, contextes. Numéro spécial de Corela (revue en ligne).

Croft, W. (en préparation). Verbs: Aspect and Argument Structure. Oxford, O.U.P. (une version provisoire est consultable sur www.unm.edu/ wcroft/WACpubs.html). 
Damourette, J. et Pichon, E. (I9I I-I936). Des Mots à la pensée. Essai de grammaire de la langue française. Paris: d'Artrey.

Demirdache, H. et Uribe-Etxebarria, M. (2002): La grammaire des prédicats spatiotemporels: temps, aspects et adverbes de temps. Dans: B. Laca (dir.), Temps et aspect. De la morphologie à l'interprétation. Saint-Denis: PUV, pp. I25-I76.

Dik, S. (1989). The Theory of Functional Grammar I, Dordrecht: Foris.

François, J. (I993). La compréhension de l'articulation chronologique des séquences narratives: un exercice de linguistique cognitive. Verbum, 1993/4: 49-70.

François, J. (2003). La Prédication verbale et les cadres prédicatifs. Louvain: Peeters.

Gosselin, L. (1996). Sémantique de la temporalité en français. Louvain-la-Neuve: Duculot.

Gosselin, L. (2005). Temporalité et modalité. Bruxelles: Duculot-De Boeck.

Gosselin, L. et Person, C. (2005). Temporalité. Dans: P. Enjalbert (dir.), Sémantique et traitement automatique du langage naturel. Paris: Hermès-Lavoisier, pp. I73-2I 3.

Gouhenheim, G. (I929). Etude sur les périphrases verbales de la langue française. Paris: Les Belles Lettres.

Gross, G. et Prandi, M. (2004). La Finalité. Bruxelles: Duculot-De Boeck.

Gross, M. (I999). Sur la définition d'auxiliaire du verbe. Langages, I 35: 8-2 I.

Heine, B. (I993). Auxiliaries. Cognitive Forces and Grammaticalization. Oxford: Oxford University Press.

Hopper, P. J. et Traugott, E. C. (1993). Grammaticalization. Cambridge: Cambridge University Press.

Jackendoff, R. (1992). Parts and Boundaries. Dans: B. Levin et S. Pinker (dir.), Lexical and Conceptual Semantics. Oxford: Blackwell, pp. 9-45.

Kamp, H. et Reyle, U. (1993). From Discourse to Logic. Introduction to Modeltheoretic Semantics of Natural Language, Formal Logic and Discourse Representation Theory. Kluwer: Dordrecht.

Klein, W. (1994). Time in Language. Londres: Routledge.

Kronning, H. (1996). Modalité, cognition et polysémie: sémantique du verbe modal 'devoir'. Acta Universitatis Upsaliensis: Uppsala.

Kronning, H. (2003). Auxiliarité, énonciation et rhématicité. Cahiers Chronos, I I: 23 I249.

Laca, B. (2004): Les catégories aspectuelles à expression périphrastique: une interprétation des apparentes 'lacunes' du français, Langue Française, I4I: 85-98.

Laca, B. (2005). Périphrases aspectuelles et temps grammatical dans les langues romanes. Dans: H. Bat-Zeev Shyldkrot et N. Le Querler (dir.), Les périphrases verbales. Amsterdam: Benjamins, pp. 47-66.

Lagae, V., Carlier, A. et Benninger, C. (2002). Présentation. Cahiers Chronos, io: i-vii.

Lambrecht, K. (2004). Un système pour l'analyse de la structure informationnelle des phrases. L'exemple des constructions clivées. Dans: J. Fernandez-Vest et S. CarterThomas (dir.), Structure informationnelle et particules énonciatives, Paris: L'Harmattan.

Lamiroy, B. (1983). Les verbes de mouvement en français et en espagnol. Lingvisticae Investigationes: Supplementa I I. Amsterdam: Benjamins.

Lamiroy, B. (1999). Auxiliaires, langues romanes et grammaticalisation. Langages, I35: $33-45$.

Leeman-Bouix, D. (1994). Grammaire du verbe français. Paris: Nathan.

Martin, R. (I97I). Temps et aspect. Essai sur l'emploi des temps narratifs en moyen français. Klincksieck: Paris.

Milner, J.-Cl. (I982). Ordres et raisons de langue. Seuil: Paris. 
Moens, M. et Steedman, M. (1988). Temporal Ontology and Temporal Reference. Computational Linguistics, I4(2): I 5-27.

Pollock, J.-Y. (1997). Langage et cognition. Introduction au programme minimaliste de la grammaire générative. Paris: PUF.

Reyle, U., Rossdeutscher, A. et Kamp, H. (2007). Ups and downs in the theory of temporal reference. Linguistics and Philosophy, 30: 565-635.

Roy, G.-R. (1976). Contribution à l'étude du syntagme verbal: étude morpho-syntaxique et statistique des coverbes. Paris: Klincksieck.

Riegel, M., Pellat, J.-Ch. et Rioul, R. (1994). Grammaire Méthodique du français. Paris: PUF.

Smith, C. (I99I). The Parameter of Aspect. Dordrecht: Kluwer.

Sundell, L.-G. (I99I). Le temps futur en français moderne. Stockholm: Almqvist \& Wiksell.

Tournadre, N. (2004). Typologie des aspects verbaux et intégration à une théorie du TAM. Bulletin de la SLP: 7-68.

Vet, C. (1987). Incorporation et grammaticalisation: verbes de mouvement et verbes de perception. Dans: B. Kampers-Manhe et C. Vet (dir.), Etudes de linguistique française offertes à Robert de Dardel par ses amis et collègues. Amsterdam: Rodopi, pp. I77-I92.

Vet, C. (I993). Conditions d'emploi et interprétation des temps futurs du français. Verbum, I993/4: 7I-84.

Vet, C. (200I). Deux cas de polysémie: le passé composé et le futur périphrastique. Dans: H. Kronning et al. (dir.), Langage et référence (Mélanges offerts à K. Jonasson). Acta Universitatis Upsaliensis: Uppsala, pp. 679-686.

Vet, C. (2002). Les adverbes de temps: décomposition lexicale et 'coercion'. Dans: B. Laca (dir.), Temps et aspect: de la morphologie à l'interprétation. Saint-Denis: PUV, pp. I79-I92.

Vet, C. (2008). Six traits sémantiques suffisent à décrire tous les temps du français. Dans: M. Birkelund, M.-B. Mosegaard Hansen et C. Norén (dir.), L'énonciation dans tous ses états (Mélanges offerts à H. Nølke). Berne: Peter Lang, pp. 45 I-47I.

Wagner, R.-L. et Pinchon, J. (I962). Grammaire du français classique et moderne. Paris: Hachette.

Wilmet, M. (1997). Grammaire Critique du français. Louvain-la-Neuve: Duculot. 